\title{
A Strategy for Optimal Operation of Hybrid AC/DC Microgrid under Different Connection Failure Scenarios
}

\author{
Van-Hai Bui, Akhtar Hussain, and Hak-Man Kim* \\ Incheon National University, Korea. \\ hmkim@inu.ac.kr
}

\begin{abstract}
This paper proposes an operation strategy to optimize the day-ahead scheduling of hybrid AC/DC microgrid (MG). The strategy schedules its resources, such as distributed generators, AC/DC battery energy storage systems to minimize the operation cost while increasing the system reliability. Moreover, an energy management system (EMS) is developed based on centralized optimization framework, which is used to optimize the $M G$ operation in grid-connected mode. On the other hand, the EMS ain s to minimize the load shedding amount in both $A C$ and DC sides in islanded mode. M this study, the operation of hybrid MG under different connection faiture scenarios is also represented. The results illustrate the effectiveness of the strdtegy for different operation modes of hybrid $A C / D C M G$.
\end{abstract}

Keywords: Energy management system, hybrid AC/DC microgrid, mixed integer linear programming, optimal microgridoptimization.

\section{Introduction}

The increasing use of renewable distributed generations (RDGs) in low voltage AC distribution network is studied due to environmental issues. In order to integrate RDGs in the distributed system, the concept of mickogrid (MG) which is defined as a small-scale power system has been developed in many countries [1,2]. Nowadays, the use of DC resources such as photovoltaics (PV) and fuel cells (FCs) have been increased in recent years, which supplies DC power to consumers. The DC/AC converters are required to connect these resources intoconventional AC system. Besides, more and more DC loads are introduced $n$ the conventional $\mathrm{AC}$ grid such as electronic lighting, electric vehicles (EVs), and information lechnology (IT) facilities, which results in the installation of the AC/DC convertersto serve DC loads. Therefore, the integration of DC resources, DC loads in the conventional AC MG, which leads to the increase in the number of DC/AC, $\mathrm{AC} / \mathrm{DC}$ converters. It will result in a more complicated system and increase the number of point of farlure, the operation losses. However, the conventional AC demands also require the existing $\mathrm{AC}$ resources. In order to take the advantage of $\mathrm{DC}$ and $\mathrm{AC}$ systems, the conoepts of hybrid AC/DC MG have been developed recently. Like the conventional AC MG, hybrid MG can operate in both grid-connected and islanded modes. In gridcomected mode, loads can be fulfilled continuously by its resources and the buying/selling power from/to the utility grid [3]. However, the MG has to fulfil its loads by its own resources in islanded mode. Load shedding strategy is implemented due to the unbalancing between supply and demands in this mode. The impacts of island load shedding strategies in autonomous MG have been analyzed in $[4,5]$. In hybrid system, the AC and DC subgrids are developed along with an interlinking converter which is used to transfer power to each other. It can directly supply both AC and DC loads. In [6], a hybrid AC/DC MG model considering the integration of RESs and plug-in hybrid electric

${ }^{*}$ Corresponding Author 
vehicles (PHEVs) has been suggested. The investigations into power-sharing issues of a hybrid MG have been introduced by [7, 8]. However, the above researches did not propose the day-ahead scheduling under connection failure states such as grid-connected, islanded modes, and the failure of interlinking converter. Therefore, this study forces on the impacts of connection failures to the day-ahead scheduling of hybrid MG. The operation mode of battery energy storage systems (BESSs), interlinking converter, the amount of load shedding, and the amount of buying/selling power are proposed in each case to operate MG in economic way.

In order to implement the proposed operation strategy for hybrid MG, an EMS is developed to operate the MG for maintaining the power balance with minimization of operation cost. The EMS architectures are grouped into centralized, decentralized, or hybrid EMS $[9,10]$. The centralized EMS architectures have been introduced by several researches. A centralized EMS (C-EMS) based on predictive control approach has been suggested by [11] for isolated MGs. In [12], a multi-stage optimization-based C-EMS for MG operation in islanded mode has been proposed. An operation strategy for optimal MGs operation in difference policies of a real-time market has been suggested by [13] based on a centralized controller. A hierarchical EMS has been proposed by [14] based on multi-agent system for multi-microgrids (MMGs) operation whereas [15] presents a nested EMS for day-ahead scheduling of MMGs. Eachor EMS has its advantages and disadvantages. According to [15], centralized energy management is the most cost efficient EMS. Therefore, in this paper, a mix integer linear program (MILP)-based model for centralized energy management has been developed for optimal hybrid MG operation. The developed model aims to minimize the operation cost. Moreover, four different connection failure scenarios are ana yzed to propose the best case for normal operation while the system can operate in the worst case.

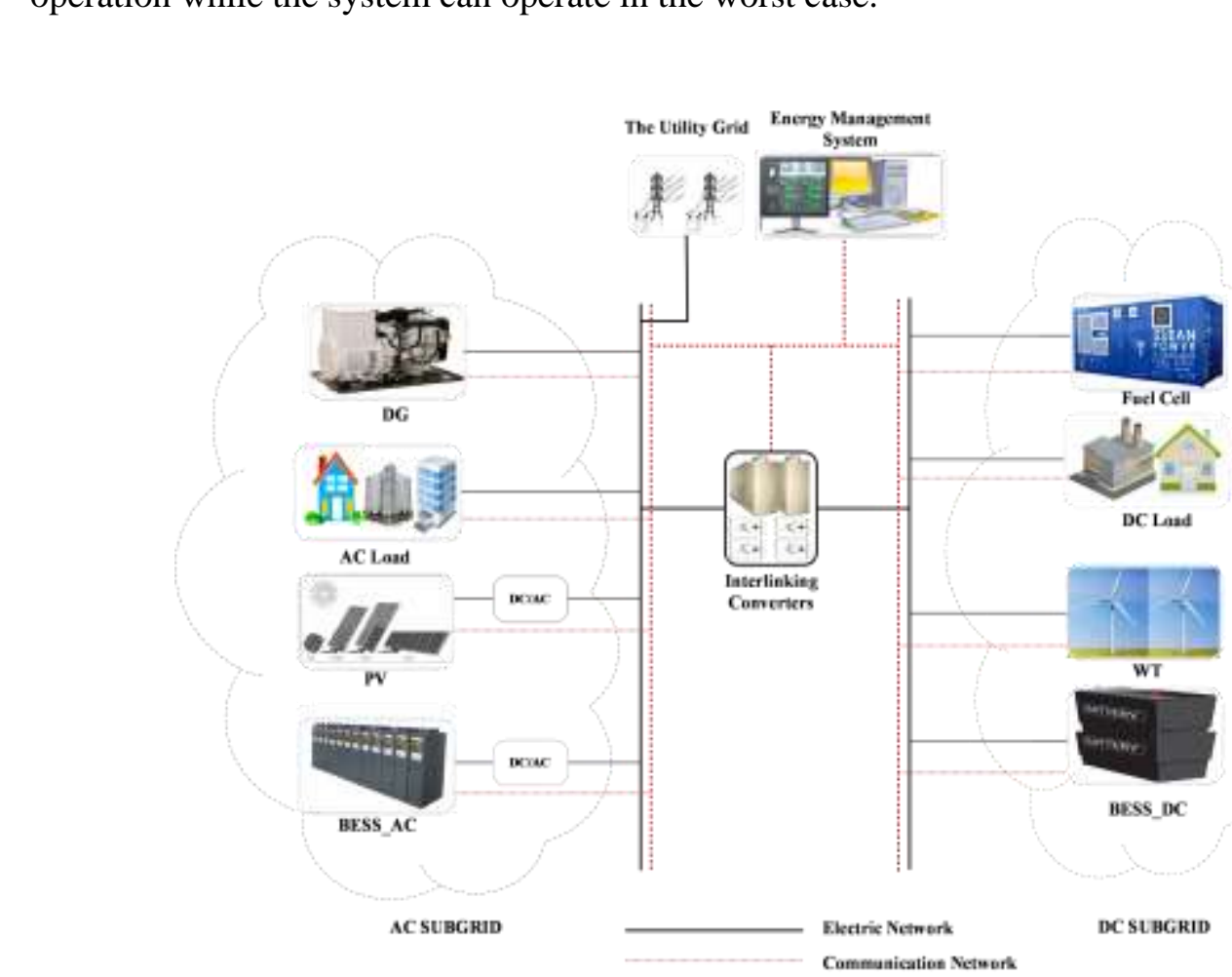

Figure 1. Test Architecture of Hybrid AC/DC Microgrid 


\section{Hybrid AC/DC Microgrid Model}

\subsection{Configuration and Components of Hybrid AC/DC Microgrid}

Figure 1 depicts an architecture of hybrid AC/DC MG. It comprises of an AC subgrid, a DC subgrid, and interlinking converters. In the AC subgrid, it is composed of DGs, PV, BESS, and AC loads. Similarly, the DC subgrid is composed of FCs, wind turbine (WT), BESS, and DC loads. In order to link AC/DC subgrids, an interlinking converter system is used. Therefore, the surplus/shortage power in both AC/DC sides can be shared to each other by using the converter system. The hybrid MG can be operated in both gridconnected and islanded modes. In normal operation, the hybrid microgrid is operated in grid-connected model. In this mode, the system can buy/sell power from/to the utility grid. On the other hand, the system has to be maintained power balance in both AC and DC sides by using its own supplies in islanded mode. Moreover, load shedding strategy could be performed to ensure the power balance in the entire system.

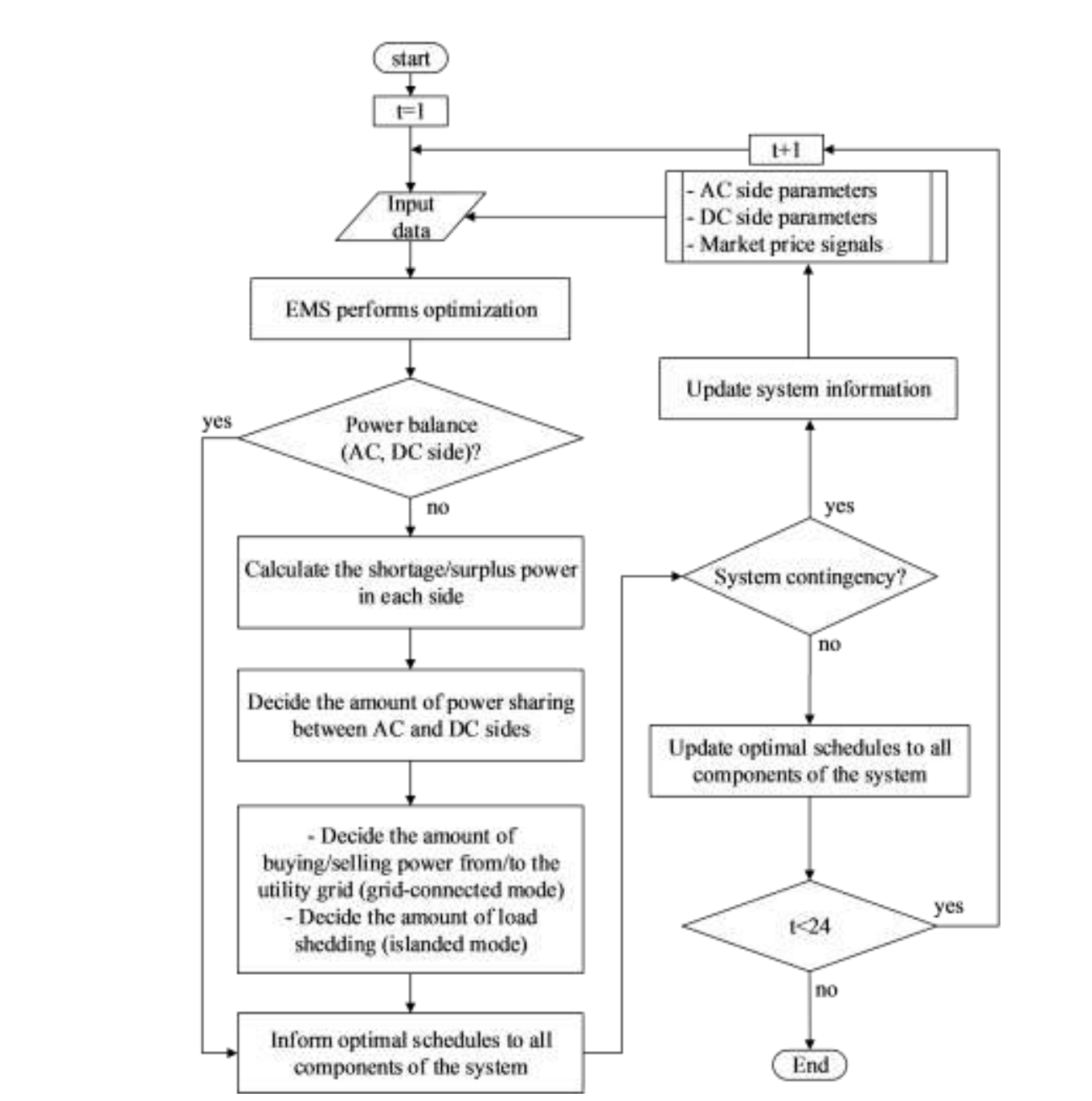

Figure 2. Proposed Operation Strategy of AC/DC Microgrid

A centralized EMS is developed to implement the operation strategy. It gathers all information from each component by using communication network. In each interval of time, the EMS decides the operation of each component in the system, the amount of purchasing/selling power from/to the utility grid, and the amount of power sharing 
between AC and DC sides. The objective of the EMS is to maintain the power balance in both of AC and DC sides while ensuring the minimization of total operation cost of the entire system.

\subsection{System Operation Algorithm}

Figure 2 depicts the flow chart related to operation of hybrid AC/DC MG. The proposed algorithm operation aims to operate entire MG along with minimization of operation cost. Firstly, the information from all components are sent to EMS via commination network, which is taken as inputs. EMS performs optimization with its received information. The amount of shortage/surplus power is determined by checking power balance in each side. In grid-connected, the shortage/surplus power is considered to exchange with other sides or the utility grid based on market price signals. On the other hand, in islanded mode, the system is operated without the utility grid. Therefore, the demand has to be fulfilled by its own supplies. In peak-time, all supplies cannot fulfil ${ }^{\star}$ their loads. Therefore, EMS has to decide the amount load shedding to maintain the power balance in the system. Finally, the optimal operation values are informed to all participating components.

In normal operation, the MG is operated based on the receiving information from EMS during one interval of time period. Whenever any failune of eonnections are detected and informed to EMS. The system information will be updated in EMS. Similarly, EMS reschedules considering updated data and informs all components along with new schedules. Figure 2 summarizes the step-by-step procedure to carry out one round of optimization.

\subsection{Mathematical Model of hybrid $\sqrt{6}$}

This section describes the developed MILP-based mathematical model of hybrid AC/DC MG. The proposed model arms tominimize the operation cost of entire MG. The cost objective function is described by (1), which includes costs associated with fuel consumption, start-up, shit down, and power exchange.

$$
\begin{aligned}
& \min \sum_{i \in I} \sum_{t \in T}\left(C_{i}^{D G} \cdot P_{j, \overrightarrow{D G}}^{\grave{D}}+r_{i, t} \cdot C_{i}^{S U_{D G}} \mathrm{Y}_{i, t} C_{i}^{S D_{D G}}\right)+\sum_{j \in J} \sum_{t \in T}\left(C_{j}^{F C} \cdot P_{j, t}^{F C}+z_{j, t} \cdot C_{j}^{S U_{F C}}+k_{j, t} \cdot C_{j}^{S D_{F C}}\right) \\
& +\sum_{t \in T}\left(P R_{t}^{b \text { lyy }} \cdot P_{t}^{B u y}-P R_{t}^{\text {Sell }} P_{t}^{\text {Sell }}\right)+\sum_{t \in T}\left(\text { pen }_{t}^{\text {Shed }_{A C}} \cdot P_{t}^{\text {Shed }_{A C}}+\text { pen }_{t}^{\text {Shed }_{D C}} \cdot P_{t}^{\text {Shed }}{ }_{D C}\right) \\
& 2 .
\end{aligned}
$$

The first termrof (1) represents the cost of DGs that is composed of operated, start-up, and shut-down costs of all DGs in AC grid. The second term describes the cost of FCs in DC grid. The third term demonstrates the cost/benefit of purchasing/selling electricity from/to the utility grid. The last term represented the penalty of shedding load in AC and DC sabgrids.

These constraints of hybrid AC/DC MG that are grouped into AC grid constraints, DC grid constraints, and interlinking converter constraints. These constraints are explained in the following sections.

\subsubsection{Constraints for AC Side}

The operated bounds and on-off mode of DGs are represented by (2), (3). Start-up status and shut-down status of DGs are defined by (4), (5), respectively.

$$
u_{i, t} \cdot P_{i}^{\mathrm{DG}_{\min }} \leq P_{i, t}^{D G} \leq u_{i, t} \cdot P_{i}^{\mathrm{DG}_{\max }} \quad \forall i \in I, t \in T
$$




$$
\begin{array}{ll}
u_{i, t} \in\{0,1\} & \forall i \in I, t \in T \\
x_{i, t}=\max \left\{\left(u_{i, t}-u_{i, t-1}\right), 0\right\} & \forall i \in I, t \in T \\
y_{i, t}=\max \left\{\left(u_{i, t-1}-u_{i, t}\right), 0\right\} & \forall i \in I, t \in T
\end{array}
$$

The balance of supply and demand is given by (6):

$$
\begin{array}{r}
P_{t}^{P V}+\sum_{i \in I} P_{i, t}^{D G}+P_{t}^{B u y}+P_{t}^{B_{A C}^{-}}+P_{t}^{\operatorname{Re} c_{D C}}+P_{t}^{\text {Shed }}{ }_{A C}=P_{t}^{\text {Load }_{A C}}+P_{t}^{\text {Sell }}+P_{t}^{B_{A C}^{+}}+P_{t}^{\text {Send }_{D C}} \\
\forall t \in T
\end{array}
$$

The constraints associated with AC-BESS include the following:

$$
\begin{aligned}
& 0 \leq P_{t}^{B_{A C}^{+}} \leq P_{B_{A C} C a p}^{C a} \cdot\left(1-S O C_{t-1}^{B_{A C}}\right) \cdot \frac{1}{1-L^{B_{A C}^{+}}} \\
& 0 \leq P_{t}^{B_{A C}^{-}} \leq P_{B_{A C}}^{C a p} \cdot S^{-C_{t-1}^{B_{A C}} \cdot\left(1-L^{B_{A C}^{-}}\right)} \\
& \operatorname{SOC}_{t}^{B_{A C}}=\operatorname{SOC}_{t-1}^{B_{A C}}-\frac{1}{P_{B_{A C}}^{C a p}} \cdot\left(\frac{1}{1-L^{B_{A C}^{-}}} \cdot P_{t}^{B_{A C}^{-}}-P_{t}^{B_{A C}^{+}}\left(1-t^{B_{A C}}\right)\right) \\
& 0 \leq \operatorname{SOC}_{t}^{B_{A C}} \leq 1
\end{aligned}
$$

Constraint (7) and (8) represent the maximum charging/discharging of AC-BESS. The SOC is updated in each interval of time according to (9). Constrain (10) enforces operated bounds of AC-BESS.

\subsubsection{Constraints for DC Sid}

Similarl, the constraints for operated bounds, on-off mode, and start-up/shut-down status of FC are represented by (11)-(14), respectively.

$$
\begin{array}{ll}
v_{j, t} \cdot P_{j}^{F C_{\text {min }}} \leq P_{j, t}^{F C} \leq v_{j, 0} P_{j}^{F C_{\text {max }}} & \forall j \in J, t \in T \\
v_{j, t} \in\{0,1\} & \forall j \in J, t \in T \\
z_{j, t}=\max \left\{\left(v_{j, t-1}-v_{j, t}\right), 0\right\} & \forall j \in J, t \in T \\
& \forall j \in J, t \in T
\end{array}
$$

Constrain (15) is power balancing for DC subgrid:

$$
P_{t}^{W T}+\sum_{j \in J} P_{j, t}^{F C}+P_{t}^{B_{D C}^{-}}+P_{t}^{\operatorname{Re}_{A C}}+P_{t}^{\text {Shed }_{D C}}=P_{t}^{\text {Load }_{D C}}+P_{t}^{B_{D C}^{+}}+P_{t}^{\text {Send }_{A C}} \quad \forall t \in T
$$

Maximum charge/discharge rate constraint of DC-BESS is given by (16), (17): 


$$
\begin{array}{ll}
0 \leq P_{t}^{B_{D C}^{+}} \leq P_{B_{D C}^{C a p}}^{C a} \cdot\left(1-S O C_{t-1}^{B_{D C}}\right) \cdot \frac{1}{1-L^{B_{D C}^{+}}} & \forall t \in T \\
0 \leq P_{t}^{B_{D C}^{-}} \leq P_{B_{D C}^{C a p}}^{C a} S^{B_{t-1}^{B_{D C}} \cdot\left(1-L^{B_{D C}^{-}}\right)} & \forall t \in T
\end{array}
$$

The amount of SOC in each interval of time is calculated by (18). Constraint (19) represents operated bounds of DC-BESS.

$$
\begin{array}{ll}
\operatorname{SOC}_{t}^{B_{D C}}=\operatorname{SOC}_{t-1}^{B_{D C}}-\frac{1}{P_{B_{D C}}^{C a}} \cdot\left(\frac{1}{1-L^{B_{D C}^{-}}} \cdot P_{t}^{B_{D C}^{-}}-P_{t}^{B_{D C}^{+}} \cdot\left(1-L^{B_{D C}^{+}}\right)\right) & \forall t \in T \\
0 \leq S O C_{t}^{B_{D C}} \leq 1 & \forall t \in T
\end{array}
$$

\subsubsection{Constraints for Interlinking Converters}

The role of interlinking converter is to share power between $\mathrm{AC}$ and $\mathrm{DC}$ sides. Equation (20) presents the constraint of maximum operation of con enter. The power balance in converter is enforced by (21), (22).

$$
\begin{aligned}
& 0 \leq P_{t}^{\operatorname{Re} c_{D C}}, P_{t}^{S e n d_{D C}}, P_{t}^{\operatorname{Re} c_{A C}}, P_{t}^{S e n d_{A C}} \leq P_{\text {Converter }}^{\text {Cap }} \\
& 0 \leq P_{t}^{\operatorname{Re} c_{D C}}=\eta_{\text {Conveter }} \cdot P_{t}^{\text {Send }_{A C}} \forall t \in T \\
& \text { 3. Numerical Results } \\
& \text { 3.1. Input Data }
\end{aligned}
$$

In this study, the proposed MHP-based model is demonstrated on a test hybrid AC/DC MG. In this system, the AC bus has 1 PV, 1 DG, 1 BESS and AC loads. Besides, DC bus has 1 WT, 1 FC, 1 BESS and DC loads. The analysis is conducted for a 24-hour scheduling horizon and each time of interval is set to be 1 hour. The market price signals, RDG outputs, and loac profiles are depicted in Figure 3.

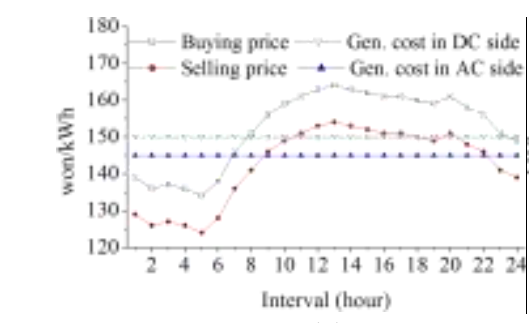

(a)

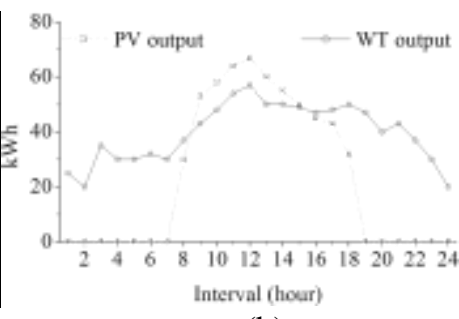

(b)

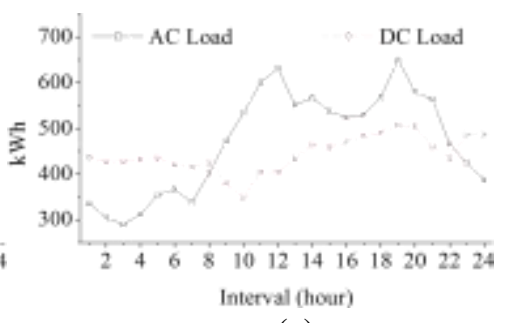

(c)

Figure 3. (a). Market Price Signals and AC/DC Side Generation cost (b). Output Power of Renewable Distributed Generations (c). AC/DC Loads

The parameters of DG, FC, and AC/DC BESSs are tabulated in table 1, 2, respectively. The capacity of interlinking converter is $500 \mathrm{kWh}$ along with $2 \%$ of transferred loss. All numerical simulation results are coded in Java and solved using the MILP solver CPLEX 12.3 [16]. 
Table 1. Generator Parameters

\begin{tabular}{|l|c|c|}
\hline \multirow{2}{*}{\multicolumn{1}{|c|}{ Parameters }} & AC Side & DC Side \\
\cline { 2 - 3 } & Diesel Generator & Fuel Cell \\
\hline Min. (kWh) & 0 & 0 \\
\hline Max. (kWh) & 500 & 400 \\
\hline Start-up cost (KRW) & 300 & 250 \\
\hline Shut-down cost (KRW) & 200 & 200 \\
\hline Operation cost (KRW)/kWh & 145 & 150 \\
\hline
\end{tabular}

Table 2. AC/DC BESSs Parameters

\begin{tabular}{|c|c|c|c|c|c|}
\hline \multirow{2}{*}{ Subgrid } & \multicolumn{5}{|c|}{ BESS parameters } \\
\cline { 2 - 6 } & $\begin{array}{c}\text { Min. } \\
(\mathbf{k W h})\end{array}$ & $\begin{array}{c}\text { Capacity } \\
(\mathbf{k W h})\end{array}$ & $\begin{array}{c}\text { Initial } \\
(\mathbf{k W h})\end{array}$ & $\begin{array}{c}\text { Char. Loss } \\
(\boldsymbol{\%})\end{array}$ & $\begin{array}{c}\text { Dis. Loss } \\
(\%)\end{array}$ \\
\hline AC & 0 & 200 & 50 & 5 & \\
\hline DC & 0 & 150 & 50 & $\mathbf{5}$ & 5 \\
\hline
\end{tabular}

Four scenarios have been considered and compared in this study as follows:

a. Normal operation (case 1): the system is in normally operation with gridconnected mode.

b. Islanded mode (case 2): the system is operated in islanded mode.

c. Failure of interlinking converters (case 3 ): there is no power sharing between $\mathrm{AC}$ and DC sides. However, AC buscan exchange power with the unity grid.

d. Failure of interlinking conventers and islunded mode (case 4): This is the worst case. The system is sêparately divided into AC, DC grids. These grids are independently operated in islanded mode.

The operation of 4 scenarios will be analyzed and compared in the following sections.

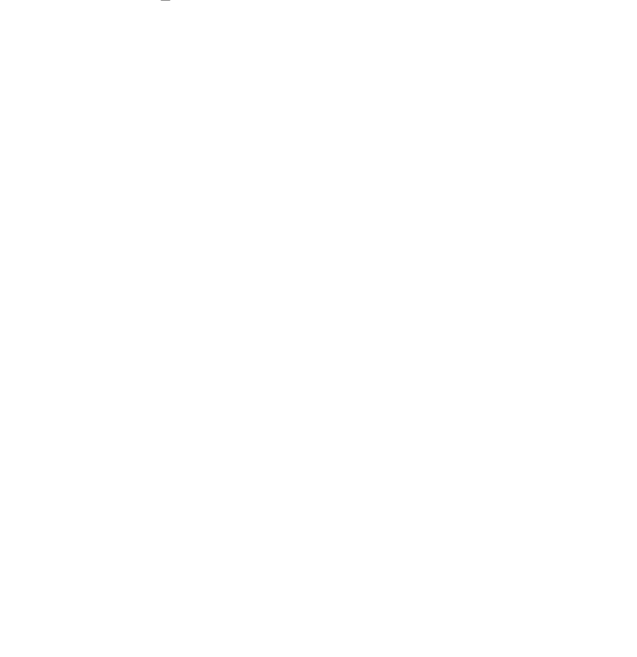




\subsection{Hybrid AC/DC MG in Normal Operation}

In this mode, a day-ahead scheduling of the hybrid AC/DC MG has been proposed with minimization of total operation cost. In each interval of time, the output power of RDG units are firstly used for reducing the operation cost. The generation power are determined based on the comparison of generation costs and market price signals. Interval $1-6$, the generation cost is less than market price, the system will buy electricity from the utility grid instead of operating generators. On the other hand, the system will sell surplus power with high marker price such as interval 10,13. Charging/discharging amount of AC/DC BESSs are decided to maximize the benefit in the system. Interval 5, in low price interval the power is fully charged to BESS and discharged on higher price intervals (10-14, 17-20). Finally, the amount of purchasing/selling power is determined to fulfill loads in the system. In this mode, the interlinking converter is used to share the cheaper resources between AC and DC sides. The day-ahead schedules for all components are summarized by Figure 4 .

Table 3. Load Shedding in the System for Difference Scenarios

\begin{tabular}{|c|c|c|c|c|c|c|c|c|}
\hline \multirow{2}{*}{$t$} & \multicolumn{3}{|c|}{ Load shedding in AC side } & \multicolumn{3}{c|}{ Load shedding in DC side } \\
\cline { 2 - 10 } & Case 1 & Case 2 & Case 3 & Case 4 & Case 1 & Case 2 & Case 3 & Case 4 \\
\hline 1 & 0 & 0 & 0 & 0 & 0 & 0 & 0 & 0 \\
\hline 2 & 0 & 0 & 0 & 0 & 0 & 0 & 0 & 0 \\
\hline 3 & 0 & 0 & 0 & 0 & 0 & 0 & 0 & 0 \\
\hline 4 & 0 & 0 & 0 & 0 & 0 & 0 & 0 & 0 \\
\hline 5 & 0 & 0 & 0 & 0 & 0 & 0 & 0 & 0 \\
\hline 6 & 0 & 0 & 0 & 0 & 0 & 0 & 0 & 0 \\
\hline 7 & 0 & 0 & 0 & 0 & 0 & 0 & 0 & 0 \\
\hline 8 & 0 & 0 & 0 & 0 & 0 & 0 & 0 & 0 \\
\hline 9 & 0 & 0 & 0 & 0 & 0 & 0 & 0 & 0 \\
\hline 10 & 0 & 0 & 0 & 0 & 0 & 0 & 0 & 0 \\
\hline 11 & 0 & 0 & 0 & 36 & 0 & 0 & 0 & 0 \\
\hline 12 & 0 & 0 & 0 & 15.36 & 0 & 0 & 0 & 0 \\
\hline 13 & 0 & 0 & 0 & 0 & 0 & 0 & 0 & 0 \\
\hline 14 & 0 & 0.38 & 0 & 12 & 0 & 14 & 14 & 14 \\
\hline 15 & 0 & 0 & 0 & 0 & 0 & 0 & 10 & 10 \\
\hline 16 & 0 & 0 & 0 & 0 & 0 & 1.42 & 22 & 0 \\
\hline 17 & 0 & 0 & 0 & 0 & 0 & 23.26 & 36 & 0 \\
\hline 18 & 0 & 36 & 0 & 36 & 0 & 38.5 & 22.5 & 0 \\
\hline 19 & 0 & 103 & 0 & 103 & 0 & 0 & 0 & 16.5 \\
\hline 20 & 0 & 0 & 0 & 0 & 0 & 0 & 0 & 64 \\
\hline 21 & 0 & 0 & 0 & 0 & 0 & 0 & 16 & 16 \\
\hline 22 & 0 & 0 & 0 & 0 & 0 & 0 & 0 & 0 \\
\hline 23 & 0 & 0 & 0 & 0 & 0 & 0 & 56 & 53.29 \\
\hline 24 & 0 & 0 & 0 & 0 & 0 & 0 & 63.29 & 66 \\
\hline
\end{tabular}




\subsection{Hybrid AC/DC MG Operation under Different Connection Failure Scenarios}

In this section, a comparison of the mentioned scenarios is represented. The load shedding amount, the operation of AC/DC BESSs, and the amount of power sharing are determined and compared in each case.

\subsubsection{Load Shedding in MG}

In islanded mode, in order to balance supply and demand, the load shedding is necessary to make power balance. This study proposed the operation strategy to minimize the amount of load shedding in AC and DC sides. The amount of load shedding in the cases are shown in table 3 . In case 1, the system operates in grid-connected mode. Hence, there is no load shedding. However, in case 2, 3, and 4, in peak-hours the system cannot fulfill its loads by its own supplies. In these cases, load shedding algorithm has to be performed to ensure power balance. As can be obtained from table 3, case 4 is the worst case for operation of the system. The amount of load shedding are decreased in case 2, 3 due to power exchange with external grids.

\subsubsection{AC/DC battery Energy Management System Operations}

The AC side BESS operation for the 4 cases are depicted by Figure 5 . It can be observed from Figure 5 that BESS has been fully charged in lower price intervals. Case 1 and 3, the AC bus is operated in grid-connected mode Therefore, the BESS is operated in economic way to maximize the system benefit as shown in Figure 5 (a), (c). On the other hand, case 2, 4, BESS is operated in islanded mode. In these cases, BESS aims to minimize the total load shedding amount as shown in Figure 5 (b), (d). In peak-hour (interval 18-22), the demand cannot befulfilled by using its own generators. Therefore, BESS is charged surplus power from off-peak intervals (13-17) and discharged to peak intervals.

Similarly, the operation of DC-BESS is demonstrated in Figure 6. In case 1and 2, DC bus is connected to AC bus by using interlinking converter. The DC bus can sell/buy the surplus/shortage power to/from AC bus for minimization of operation cost. Charging/discharging amount of BESS is depicted in Figure 6 (a), (b). In case 3, 4, the DC bus is in autonomous mode. The BESS is operated to maintain the power balance in the DC grid Charging/discharging amount of BESS is shown in Figure 6 (c), (d).

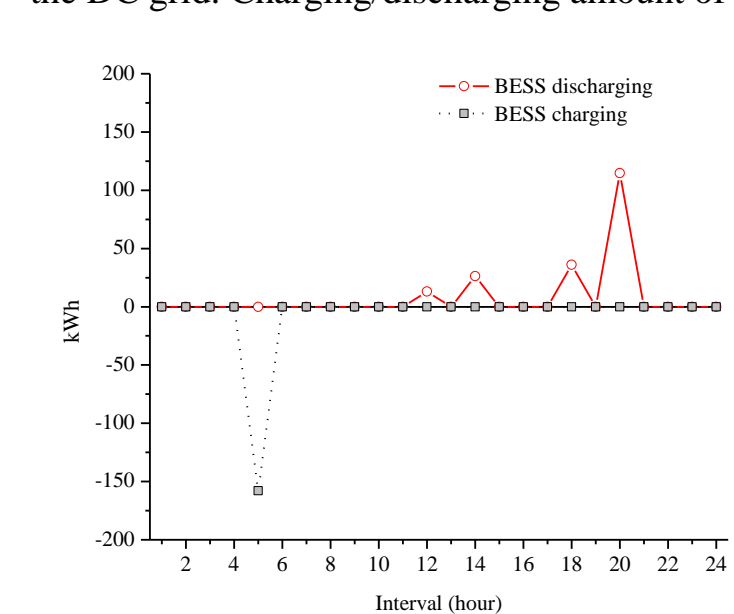

(a) Case 1

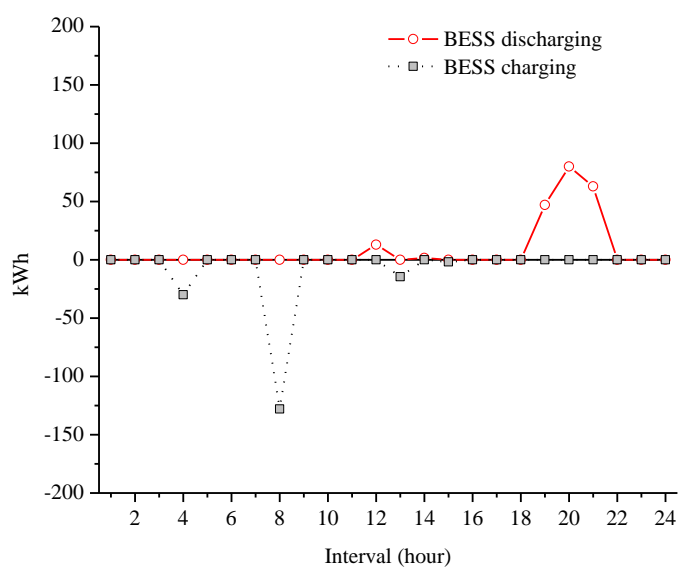

(b) Case 2 


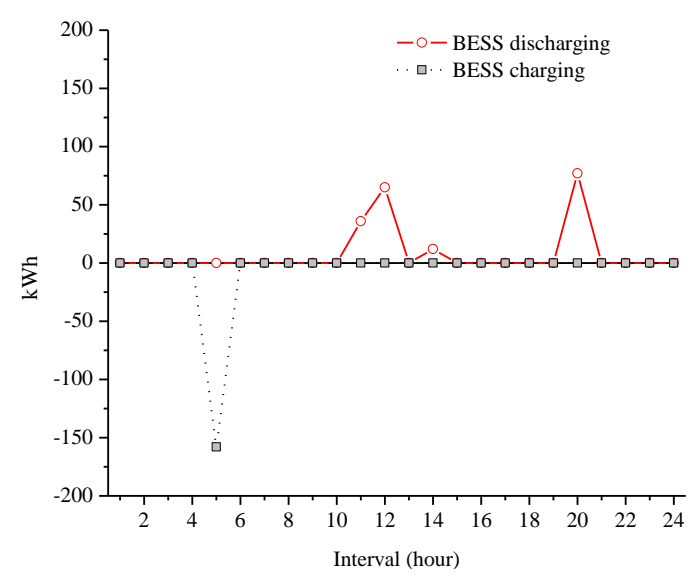

(c) Case 3

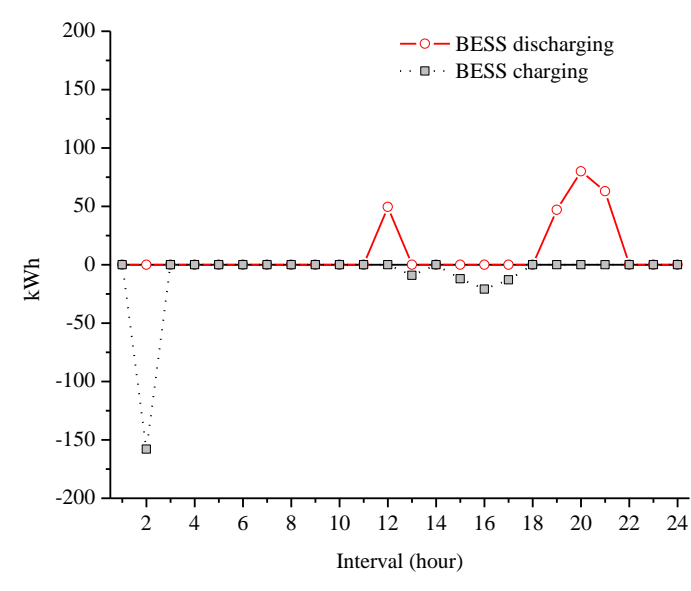

(d) Case 4

Figure 5. AC Side Battery Energy Management System Operation

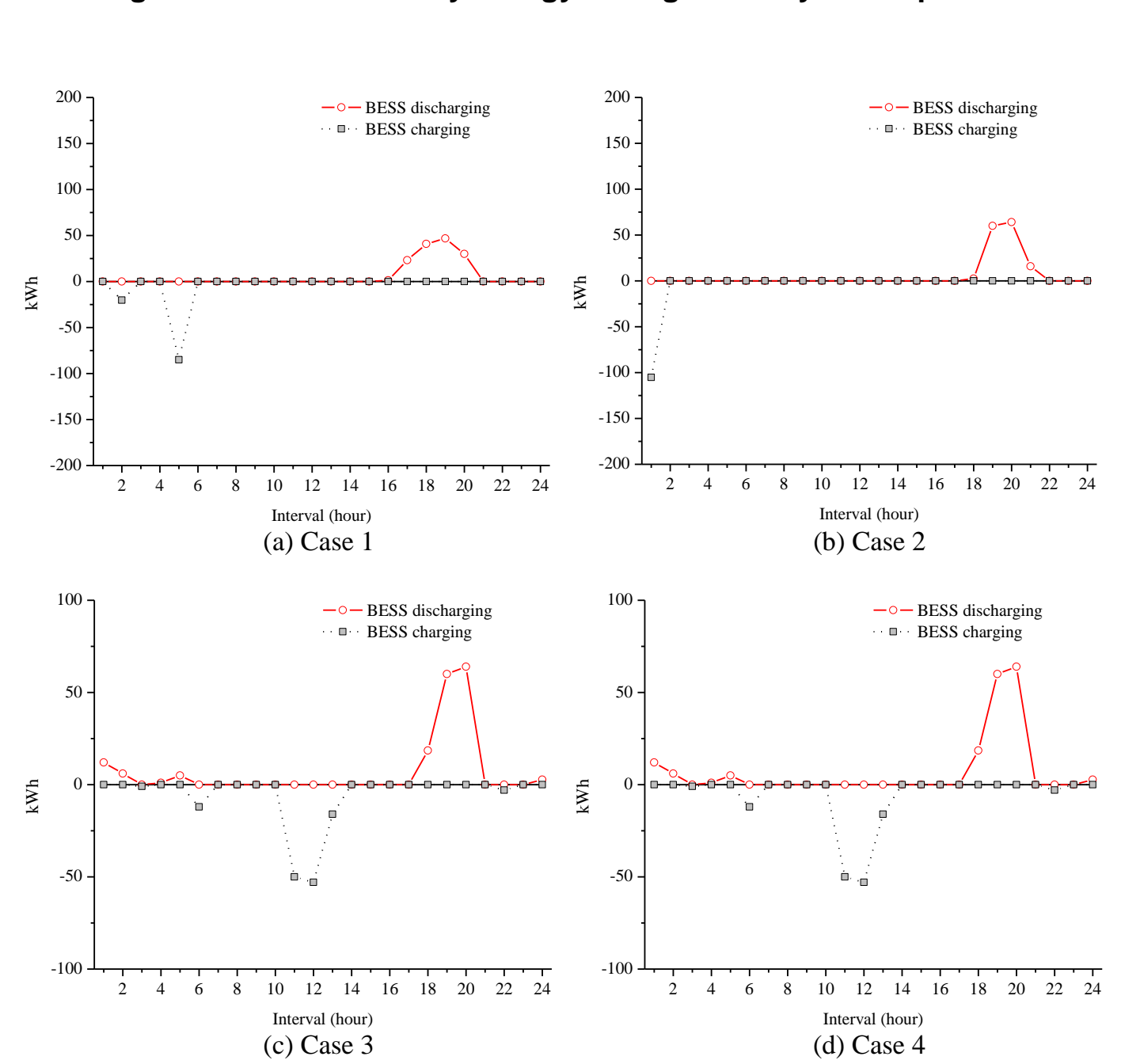

Figure 6. DC Side Battery Energy Management System Operation 


\subsubsection{Power Sharing by Using Interlinking Converters}

The amount of power sharing is shown in Figure 7. In grid-connected mode (case 1), the amount of power sharing is decided to share the cheaper resources for minimization of total operation cost. In case 2, the hybrid AC/DC MG is operated in islanded mode. The system cannot exchange power with the utility grid. Therefore, the amount of power sharing is decreased to minimize the load shedding amount as well as operation cost, as illustrated in Figure 7 (b). In case 3 and 4, the connection between AC and DC sides is broken. Hence, the amount of power sharing is zero, as shown in Figure 7 (c).

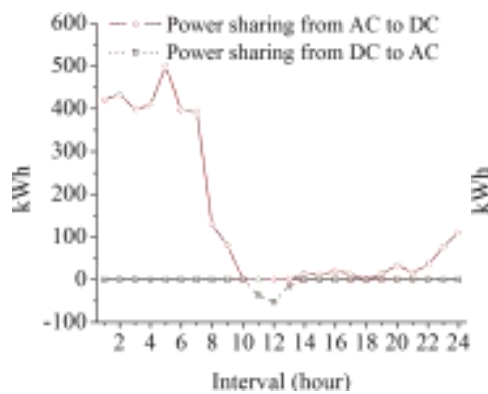

(a) Case 1

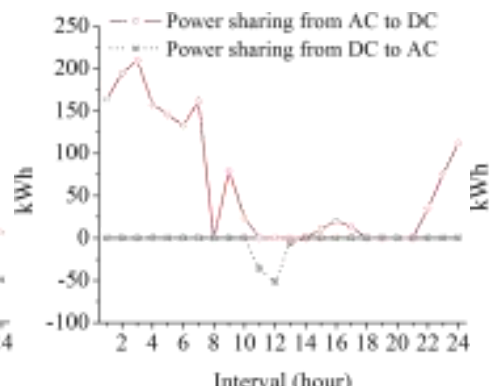

(b) Case 2

( $)$ (bsing 2

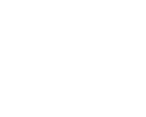

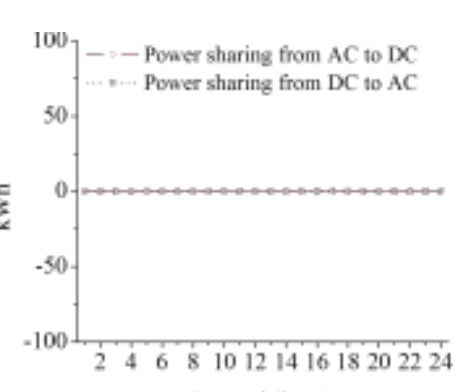

enval (hour)

Figure 7. Power Sharing by Using Interlinking Converter

\section{Conclusion}

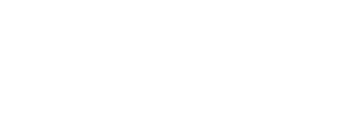

In this study, a strategy for optimal energy management in hybrid AC/DC MG has been proposed. In grid-connected mode, the test system is capable of trading surplus/deficit of electricity with the utility grid. The AC and DC subgrids can share theirs resources by using interlinking converters. The deyeloped EMS is to minimize the operation cost of the entire network while reducing the amount of load shedding in islanded mode. Moreover, the fffects on the system of different connection failures have also been analyzed and compared The amount of load shedding, the AC/DC BESS operations, and the amount of powe sharing are compared in each case of failure. By the comparison of four different scenarios, the best operation mode is chosen for normal operation. In failure cases, the operation mode of BESSs and interlinking converters are proposed for minimization of operation cost as well as the amount of load shedding. Finally, the results indicated that the system are able to operate even in the worst case in economic way by using the proposed operation strategy. 


\section{Nomenclature}

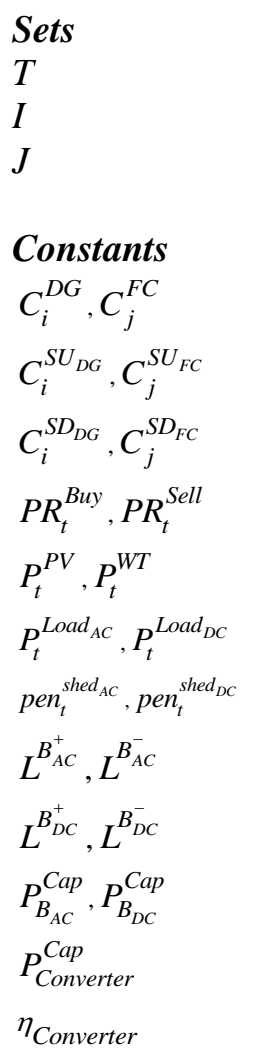

\section{Variables}

$u_{i, t}, v_{j, t}$

$P_{i, t}^{D G}, P_{j, t}^{F C}$

$P_{t}^{\text {Buy }}, P_{t}^{\text {Sell }}$

$P_{t}^{B_{A C}^{+}}, P_{t}^{B_{A C}^{-}}$

$P_{t}^{B_{D C}^{+}}, P_{t}^{B_{D C}^{-}}$

$P_{t}^{\operatorname{Re} c_{D C}}, P_{t}^{\text {Send }_{D C}}$

$P_{t}^{\operatorname{Re} c_{A C}}, P_{t}^{\operatorname{Send} A_{A C}}$

$P_{t}^{\text {Shed }_{A C}}, P_{t}^{\text {Shed }}{ }^{D C}$

$S O C+C_{A C}^{B_{D C}}$
Set of time intervals.

Set of diesel generations.

Set of fuel cells.

Production cost of DG unit i, FC unit j.

Start-up cost of DG unit i, FC unit $j$.

Shut-down cost of DG unit i, FC unit j.

Buying/selling price at $\mathrm{t}$.

Forecasted output of PV cell \& wind turbine.

AC, DC load amount at t.

Penalty for AC, DC load shedding anountat.

Losses for charging/discharging of AC BESS

Losses for charging/discharging of DC BESS.

Capacity of AC and DC BESSs

Capacity of interlinking converter

Efficiency of inferlinking converters

On or offmode of DG unit $i$ and FC unit $j$ at $t$.

Generation amoun of DG unit $i$ and FC unit $j$ at $t$.

Buying/selling power from/to the utility grid at $t$.

Chargingdischarging power of AC BESS at $t$.

Chrarging/discharging power of DC BESS at $t$.

Receiving/sending power from/to DC subgrid at t.

Receiving/sending power from/to AC subgrid at t.

Load shedding amount in AC and DC subgrids at $\mathrm{t}$.

State of charge for AC BESS and DC BESS at t.

\section{Acknowledgements}

This work was supported by the Korea Institute of Energy Technology Evaluation and Planning (KETEP) and the Ministry of Trade, Industry \& Energy (MOTIE) of the Republic of Korea (No. 20168530050030). 


\section{References}

[1] [1] R. H. Lasseter and P. Paigi, "Microgrid: A conceptual solution," in Proc. IEEE Power Electron. Spec. Conf., (2004), Jun., pp. 4285-4290.

[2] R. H. Lasseter, "MicroGrids," in Proc. IEEE Power Eng. Soc., (2002), pp. 305-308.

[3] A. Hussain, V. H. Bui, H. M. Kim, "Robust optimization-based scheduling of multi-microgrids considering uncertainties," Energies, vol. 9, no. 4, (2016), Apr., pp. 278.

[4] H.-M. Kim, Y. Lim, and T. Kinoshita, "An intelligent multiagent system for autonomous microgrid operation," Energies, vol. 5, no. 9, (2012), Sept., pp. 3347-3362.

[5] Y. Lim and H.-M. Kim, and T. Kinoshita, "Distributed load-shedding system for agent-based autonomous microgrid operations," Energies, vol. 7, no. 1, (2014), pp. 385-401.

[6] P. T. Baboli, S. Bahramara, M. P. Moghaddam, and M. R. Haghifam, "A mixed-integer linear model for optimal operation of hybrid AC-DC microgrid considering Renewable Energy Resources and PHEVs," In Power Tech., IEEE Eindhoven, (2015), Jun., pp. 1-5.

[7] P. C. Loh, D. Li, Y. K. Chai, and F. Blaabjerg, "Autonomous operation of hybrid microgrid with AC and DC subgrids," IEEE trans. on power electronics, vol. 28, no. 5, (2013), pp.2214-2223.

[8] N. Eghtedarpour and E. Farjah, "Power control and management in a hybrid AC/DC microgrid," IEEE Trans. on Smart Grid, vol. 5, no. 3, (2014), pp.1494-1505.

[9] F. Katiraei, R. Iravani, N. Hatziargyriouand A. Dimeas, "Microgrid management," IEEE Power Energy Mag., vol. 6, no. 3, (2008), May, pp. 54-65.

[10] W. Su and J. Wang, "Energy Management Systems in Microgrid Operations, The Electricity Journal, vol. 25, no. 8, (2012), Oct., pp. 45-60.

[11] D. E. Olivares, C. A. Canizares, and M. Kazerani, "A centralized energy managenent system for isolated microgrids,” IEEE Trans. Smart Grid, vol. 5, no. 4, (2014), Jul., pp. 1864-1875.

[12] D. Olivares, C. Canizares and M. Kazerani, "A centralized optimal energy management system for microgrids,”In Proc. IEEE/PES Gen. Meet., (2011), Jul., pp. $1-6$.

[13] A. G. Tsikalakis and N. D. Hatziargyriou, "Centralized control for optimizing microgrids operation," In Power and Energy Society General Meet. IEEE, (2011), Jul., pp.4-

[14] V. H. Bui, A. Hussain, and H. M. Kim, "A mulitiagent-based hierarchical energy management strategy for multi-microgrids considering adjustable power and demand response," IEEE Trans. on Smart Grid, accepted, (2016), pp. 1-10.

[15] A. Hussain, V. H. Bui, and H. M. Kim, A resilient an privacy-preserving energy management strategy for networked microgrids," IEEE Trans. on Smart Grid, accepted, (2016), pp. 1-12.

[16] IBM ILOG CPLEX V12.1 User's Manual,fo CPLEX 2009, CPLEX Division, ILOG, Incline Village, NV, USA, (2009).
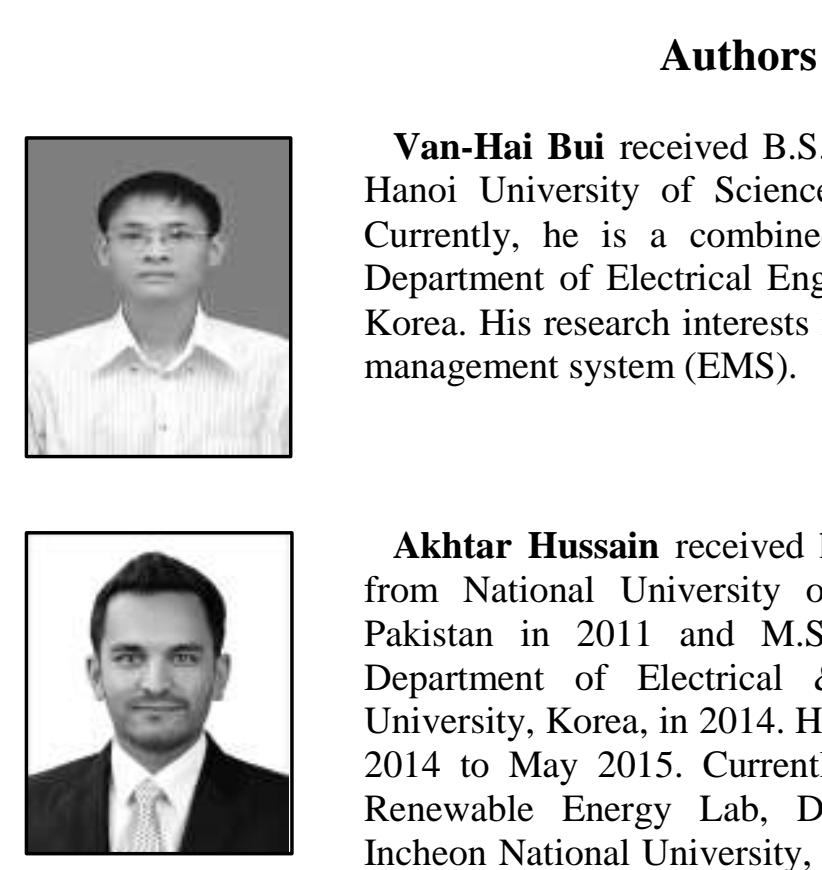

Van-Hài Bui received B.S. degree in Electrical Engineering from Hanoi University of Science and Technology, Vietnam in 2013. Currently, he is a combined Master and $\mathrm{Ph}$. D. student in the Department of Electrical Engineering, Incheon National University, Kogea. His research interests include microgrid operation and energy management system (EMS).

Akhtar Hussain received his B.E degree in Telecommunications from National University of Sciences and Technology (NUST) Pakistan in 2011 and M.S in Electrical Power Systems from Department of Electrical \& Electronics Engineering, Myongji University, Korea, in 2014. He worked for SANION, Korea from Jan 2014 to May 2015. Currently he is a Ph.D. student in Power \& Renewable Energy Lab, Department of Electrical Engineering, Incheon National University, Korea. His research interests are power system automation and protection, smart grids, operation of microgrids, and energy management in microgrids. 


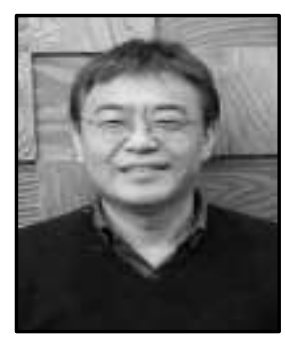

Hak-Man Kim received his first Ph.D. degree in Electrical Engineering from Sungkyunkwan University, Korea in 1998 and received his second $\mathrm{Ph}$. D. degree in Information Sciences from Tohoku University, Japan, in 2011, respectively. He worked for Korea Electrotechnology Research Institute (KERI), Korea from Oct. 1996 to Feb. 2008. Currently, he is a professor in the Department of Electrical Engineering, Incheon National University, Korea. His research interests include microgrid operation \& control and DC power systems.

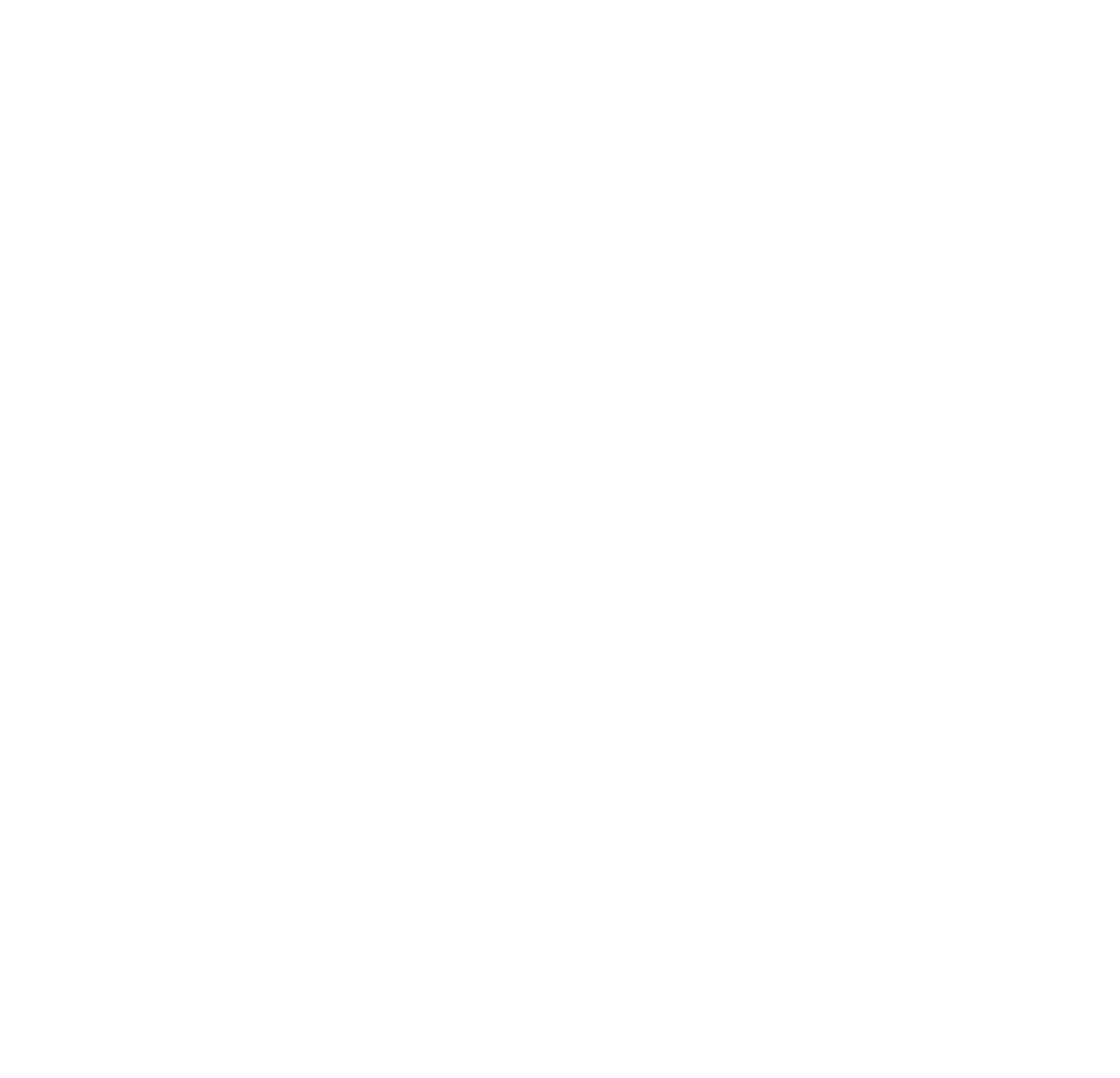

